\title{
Electron Line Broadening in Plasmas: Resolution of the quantum vs. semiclassical calculations puzzle
}

\author{
by \\ S. Alexiou and R. W.Lee
}

${ }^{1}$ Lawrence Livermore National Laboratory, PO Box 808 Livermore CA 94551

\section{May 172004}

Disclaimer:

This document was prepared as an account of work sponsored by an agency of the United States Government. Neither the United States Government nor the University of California nor any of their employees, makes any warranty, express or implied, or assumes any legal liability or responsibility for the accuracy, completeness, or usefulness of any information, apparatus, product, or process disclosed, or represents that its use would not infringe privately owned rights. Reference herein to any specific commercial product, process, or service by trade name, trademark, manufacturer, or otherwise, does not necessarily constitute or imply its endorsement, recommendation, or favoring by the United States Government or the University of California. The views and opinions of authors expressed herein do not necessarily state or reflect those of the United States Government or the University of California, and shall not be used for advertising or product endorsement purposes.

Auspices

This work was performed under the auspices of the U.S. Department of Energy by University of California, Lawrence Livermore National Laboratory under Contract W-7405-Eng-48. 


\title{
Electron Line Broadening in Plasmas: Resolution of the quantum vs. semiclassical calculations puzzle
}

\author{
S. Alexiou and R.W. Lee \\ L-399, P.O.Box 808, Lawrence Livermore National Laboratory, CA 94550,USA
}

\begin{abstract}
Puzzling discrepancies between on the one hand quantum mechanical(QM) electron impact calculations of isolated ion lines and on the other hand experimental data and nonperturbative semiclassical(SC) calculations are reviewed. The origin of these discrepancies was a standard linebroadening literature estimate of the wavefunction extent. The nonperturbative semiclassical calculations are further improved by dropping the long-range approximation and allowing penetrating collisions. This results in excellent agreement with fully quantal calculations for the case of the BIII $2 \mathrm{~s}-2 \mathrm{p}$ line. On the other hand the standard perturbative semiclassical method is inadequate even in this particular example, where perturbation theory is valid. Further, the assumption of neglecting the back-reaction in semiclassical calculations is examined.
\end{abstract}

\section{INTRODUCTION}

Isolated lines from ion emitters provide test of electron Stark broadening(SB) alone: Because electron SB is generally small, and because ion SB is generally also very small, as we will see, ions are usually impact ${ }^{1}$. In that case, the key factor is the imaginary exponentials of the emitter-perturber interaction in the interaction picture, e.g. if $\mathrm{V}(\mathrm{t})$ is the interaction in the Schrödinger picture, we have integrals of the form $\int d t V(t) e^{i \omega_{\alpha \alpha^{\prime}} t}$ where $\omega_{\alpha \alpha^{\prime}}$ is the energy difference in angular frequency units between an upper or lower level state $\alpha$ and a perturbing state $\alpha^{\prime}$.

For ion lines, electrons and ions act differently [1]: Electrons come closer to the emitter, but stay less; ions do not come as close, but stay longer. The upshot is that to second order in perturbation theory and for a dipole interaction the electron and ion contributions are equal for a given velocity if $\omega_{\alpha \alpha^{\prime}}=0$ (of course the ion velocity distribution favors much smaller velocities and the final width will not be the same). However, to achieve this equal contribution, electrons rely on some

1) Recall that at a given set of plasma parameters, ion impact is the largest possible contribution the ions could make, i.e. if we were to compute the width by using the ion impact approximation for ions, we would in no case be underestimating the width. This follows from the impact approximation being linear in density, whereas all other regimes have a sublinear dependence. 


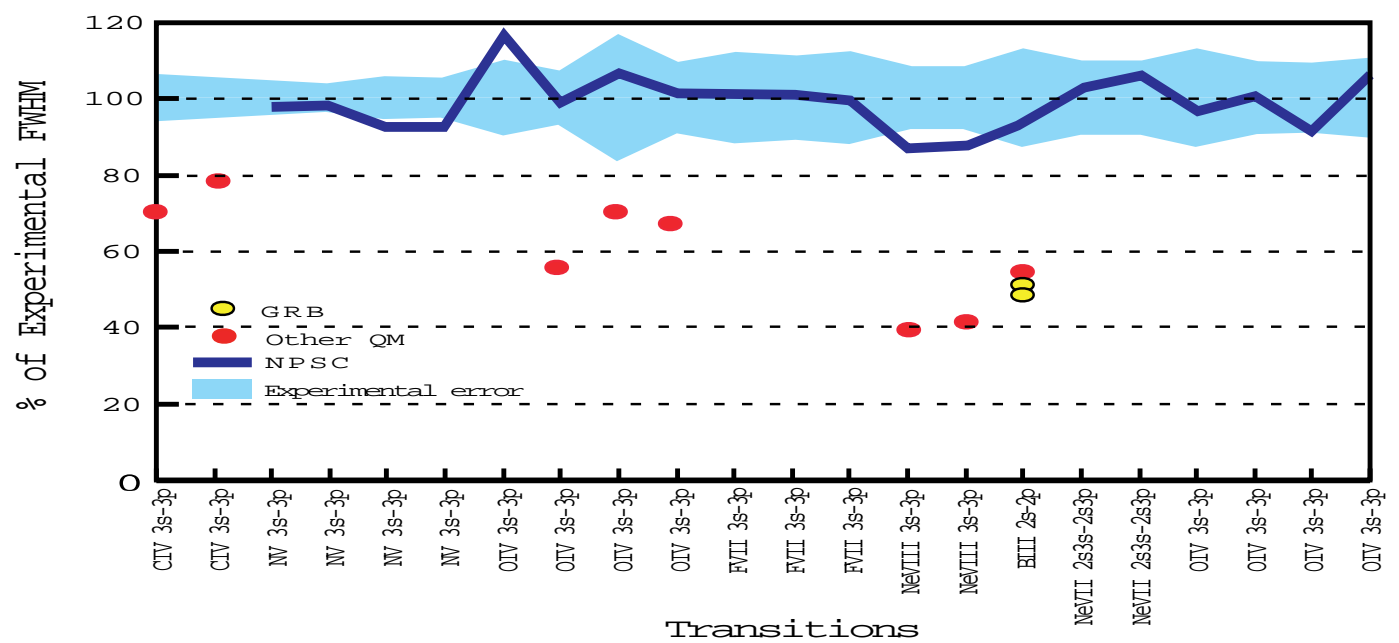

FIGURE 1. Theory vs. Experiment

close, short-lived encounters, while ions rely on weaker but longer lasting collisions. When $\omega_{\alpha \alpha^{\prime}} \neq 0$ only short times contribute. This is a killer for ions who now can only use a small portion of a long time interval. Electrons are not affected as much, since they relied on a short time interval anyway.

\section{SC, QM METHODS AND EXPERIMENTS}

A recent improvement in semiclassical(SC) calculations was the Nonperturbative semiclassical method(NPSC) [2]. Briefly, collisions are separated by a minimum impact parameter(IP) $\rho_{\min }(v)$ into those that may and those that may not be treated in SC. Those that may not be treated in SC either have a de Broglie wavelength larger than $\rho / A$, where $\rho$ is the IP and $\mathrm{A}$ is a parameter, set equal to 1 in NPSC(basically this says how much smaller than $\rho$ the de Broglie wavelength has to be in order for SC to be valid), or an IP smaller than the emitter wavefunction extent(WFE) for the upper level in question, in which case the long-range approximation used is not valid. For non SC-treatable collisions, a unitarity-based error bound is returned, i.e. the contribution of the non-SC part of phase space is bound by unitarity. Unlike PR, which had to generally increase $\rho_{\min }(v)$ substantially to ensure that unitarity is preserved by a PR expansion, NPSC automatically satisfies unitarity. This in turn decreased the error bound and increased the part of phase space whose width contribution was accurately computed.

Fig. 1 shows the highest quality experimental data from the Bochum gas-liner pinch [3,4] as of 1996 for a number of measured isolated lines. The plasma parameters are determined by Thomson Scattering(TS). The solid graph and grey area is the ratio of the experimental to the theoretical NPSC widths and associated error bars, respectively. The isolated dots represent the ratio of the experimental width to the width computed by fully quantum mechanical(QM) close-coupling $(\mathrm{CC})$ methods [5-7]. All calculations described here refer to Ref [4]. 


\section{QM CRITIQUE OF NPSC AND EXPERIMENTS}

In [6] it was proposed that experiments are wrong because they are affected by undiagnosed turbulence and NPSC calculations are wrong because of the cutoffs used. Specifically, it was argued that parameter A for the de Broglie cutoff should be set to $2 \pi$ and that the wavefunction(WF) extent cutoff should read "the distance of closest approach should be larger than the relevant WFE", rather than the IP being larger than the WFE, as the electron is attracted by the ionic charge. Of these proposals the first is by far the most drastic one. If NPSC were to set $A=2.3$, it would recover the results of Ref [5], which are still larger than Ref. [6]. This is a very radical challenge to the accepted, standard understanding of electron impact $\mathrm{SB}$, as it impacts on a large number of issues, for example:

Do we have any reliable experiments? The gas-liner pinch experiments are among the highest quality experiments ever in the field. In critical reviews [8], the gasliner pinch experiments get a "C" rating, which implies an error bar insufficient to bring them in agreement with with the quantal results. In other words if Ref. [6] is correct, then the rating of the gas-liner experiments in Ref [8] is erroneous.

The de Broglie cutoff proposal is also a drastic change. Standard (PR) theory had it that the $\rho_{\text {min }}$ cutoff for hydrogen lines, namely $n^{2} \lambda$, with $\lambda$ the reduced de Broglie wavelength and $n$ the upper level principal quantum number satisfied the de Broglie requirement, as it was "considerably larger" [9] than the reduced de Broglie wavelength and calculations were performed for $L y_{\alpha}$, where $n^{2}=4<2 \pi$. Hence even though for hydrogenic lines $\rho_{\min }$ is not as critical as for nonhydrogenic ones(unless it becomes comparable to the maximum IP, i.e. high densities and/or low temperatures), the issues raised here are relevant for $\mathrm{H}$-like lines and as we will see they are also relevant for neutral emitters and also ion perturbers. Literature [10] had always used the NPSC cutoffs. NPSC did not accept this critique, with the objections summarized in Ref [11]. In addition, satisfying almost all requests except the $2 \pi$ factor still gave widths larger than Ref [6].

\section{TESTING THE DE BROGLIE CUTOFF}

To test the choice of A, we performed calculations [13] using the CoulombBethe(CB) approximation. This has the advantages of being fully quantummechanical, simple and preserves the long-range approximation. Hence we can compare PR SC(and NPSC for large partial waves where unitarity issues are not relevant) to fully quantal methods with all other things(i.e. approximations) being equal. An initial check revealed no deviations in the line width contribution as a function of partial wave $l$, as in Fig 2(A).

However, checking the cross sections ${ }^{2}$ as a function of partial wave at an energy close to the average gave much larger cross sections for high partial waves

2) NPSC does not employ cross sections, but instead computes S-matrices; It was extended to compute cross-sections for the purposes of this comparison. 

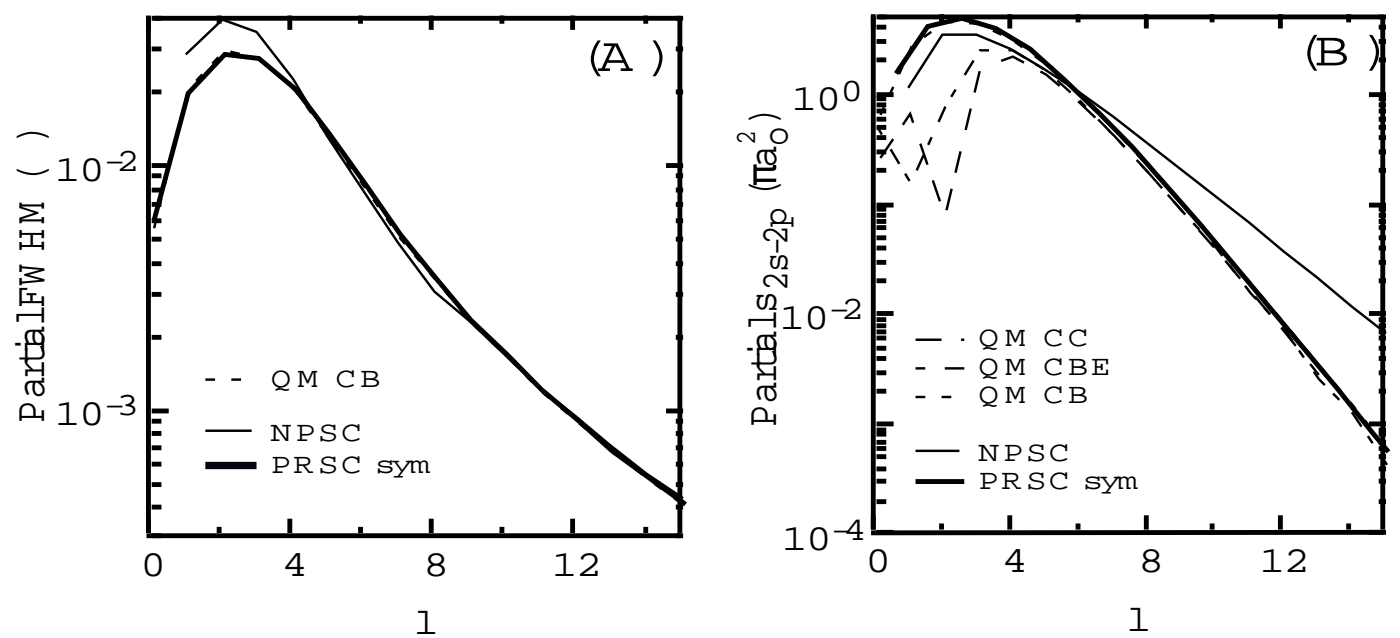

FIGURE 2. NPSC vs. Coulomb-Bethe

than all quantal methods(Fig. 2(B)). When the PR SC cross-sections were symmetrized(PRSC sym in the graph), excellent agreement was obtained( $\leq 6 \%$ for $\mathrm{s}$ and $\mathrm{p}$ waves and better for all partial waves below 10). We note that high partial waves $(>6)$ are completely insignificant to the line width. CBE refers to CoulombBorn with Exchange in Fig.(2B). Thus, this check is a clear indication that at least in this particular case the de Broglie cutoff is not the problem. On the other hand, it does hint that back-reaction may be an issue in SC.

\section{NPSC EVOLVES: NPSCII}

In the last year, NPSC has improved by dropping the long-range approximation and using the full Coulomb interaction instead. We refer to this improved version as NPSCII. NPSC needed an estimate of the WFE, in order to estimate at what point corrections associated with the breakdown of the long-range approximation to the full multipole expansion enter into play. This was taken after Griem [10], i.e. the WFE was estimated as $n^{2} a_{0} / Z$ with $n$ the upper level principal quantum number, $a_{0}$ the Bohr radius and $Z$ the spectroscopic charge number. NPSCII does not need or employ any WFE cutoff and only uses $\rho_{\min }(v)=A \hbar / m v$. The price to be paid is that matrix elements are no longer enough, but instead WF information is required(Standard SC theory was simple in that matrix elements alone were used). The minimal sufficient such information is what we call below "correction factors". Technically, this means that the computation can proceed as before, except that now the $\lambda \operatorname{th}(=1$ for dipole, 2 for quadrupole etc.) multipole matrix element is multiplied by the correction factor $C_{\lambda}$. For a dipole or quadrupole matrix element between states with principal quantum numbers $(\mathrm{n}, \mathrm{l})$ and $\left(n^{\prime}, l^{\prime}\right), C_{\lambda}$ is defined as:

$$
C_{\lambda}\left(R ; n, l, n^{\prime}, l^{\prime}\right)=\frac{\int_{0}^{R} P_{n l}(r) P_{n^{\prime} l^{\prime}}(r) r^{\lambda} d r}{\int_{0}^{\infty} P_{n l}(r) P_{n^{\prime} l^{\prime}}(r) r^{\lambda} d r}+R^{2 \lambda+1} \frac{\int_{R}^{\infty} d r P_{n l}(r) P_{n^{\prime} l^{\prime}}(r) r^{-(\lambda+1)}}{\int_{0}^{\infty} P_{n l}(r) P_{n^{\prime} l^{\prime}}(r) r^{\lambda} d r}
$$



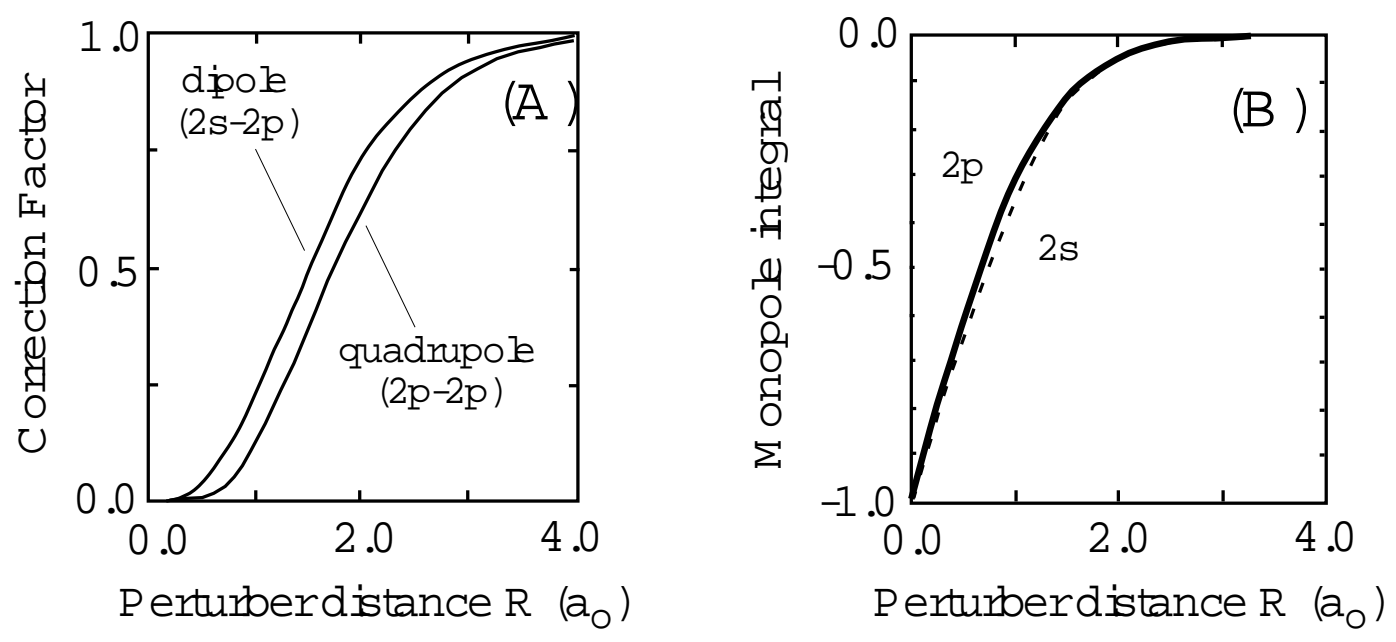

FIGURE 3. Correction Factors and Monopoles

with $\mathrm{R}(\mathrm{t})$ the position of the perturber at time $\mathrm{t}$ and $\lambda$ the multipole or$\operatorname{der}(1=$ dipole, $2=$ quadrupole $) . C_{\lambda}$ arises from the fact that the needed matrix elements of the Coulomb interaction are expressed as

$$
\left\langle i\left|\frac{1}{|\mathbf{r}-\mathbf{R}(\mathbf{t})|}-\frac{1}{R(t)}\right| j\right\rangle=\sum_{l=0}^{\infty} \sum_{m=-l}^{l} \frac{4 \pi}{2 l+1} \frac{r_{<}^{l}}{r_{>}^{l+1}} Y_{l m}^{*}(\Omega) Y_{l m}(\Omega)-R^{-1} \delta_{i j}
$$

$C_{\lambda}$ may be thought of as approximately the ratio of the value of the effective multipole matrix element when the perturber electron is at $\mathrm{R}(\mathrm{t})$ to the corresponding matrix element when the perturber electron is at infinity(or completely outside the WFE). As Fig.3(A) shows over $50 \%$ of the dipole matrix element comes from radii beyond $1.33 a_{0}$, i.e. the $n^{2} a_{0} / Z$ cutoff, with the situation being worse for the quadrupole matrix element.

The standard cutoff is based on the association of the electron radius in the Bohr picture to the WFE and is appropriate at sufficiently high principal quantum numbers. In cases of practical interest for the SB of isolated ion lines, the standard WFE cutoff may be seriously inaccurate. Note that this also affects neutral lines and, in principle, SB by ions entering the WFE. This finding is different from the second point in Ref. [6] in that WFE is penetrated not only close to the perihelion, but even on the asymptote. In addition, the monopole contribution

$$
\frac{e^{2} Z_{p e r t}}{4 \pi \epsilon_{0} R} M(R), M(R)=\int_{R}^{\infty} \frac{P_{n l}(r) P_{n^{\prime} l^{\prime}}(r)}{a_{0} r / R} d r-\int_{R}^{\infty} P_{n l}(r) P_{n^{\prime} l^{\prime}}(r) d r
$$

was added. $Z_{\text {pert }}$ is the perturber charge and the integrals are in atomic units. This is diagonal in all quantum numbers except the principal quantum number. $M(R)$ is displayed in Fig.3(B) and turns out to be a small effect as far as the width is concerned and mainly cancells a part of the quadrupole contribution. 


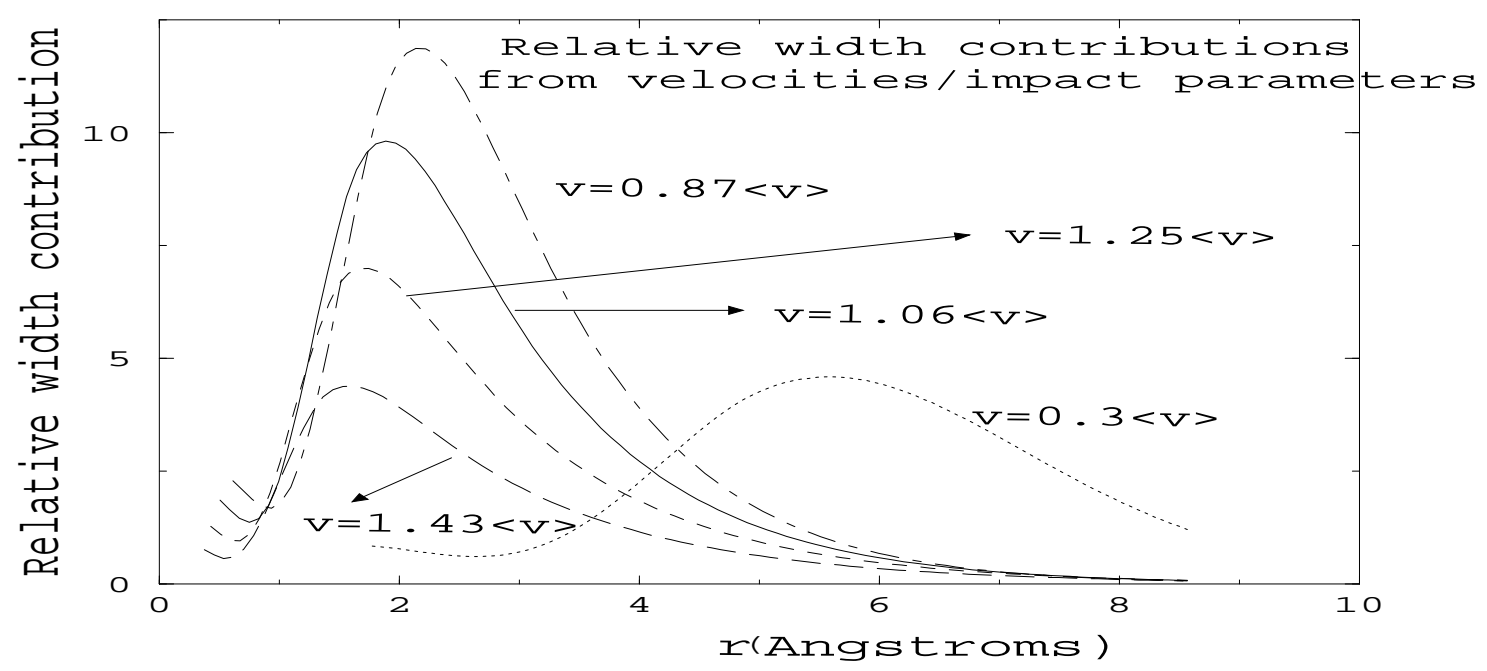

FIGURE 4. Velocities and IPs giving the dominant contribution to the width

The NPSCII FWHM value is $10 \%$ larger than the R-matrix calculations of [5] and $5 \%$ smaller than the value of Ref. [6], i.e. between the two CC calculations. This assumes a 0 strong collision estimate from the s-wave, which is probably very small, judging from QM calculations. However, the unitarity-based error bound is $20 \%$.

\section{BACK-REACTION ISSUES}

As already hinted, a major issue could be energy transfer considerations and their effect on the SC trajectory. Energy transfers associated with collisionallyinduced transitions of the radiating electron can in the case of isolated lines be comparable to the kinetic energy of the perturbing electrons. SC always assumed that the perturber trajectory is unaffected by such processes. Such collisionallyinduced transitions however, occur in pairs in the SB problem, as we are interested in processes where the radiating electron ends up in the same state it started. One way these processes will not affect the SC trajectory is if a pair of exitationdeexcitation to the initial state occurs on a time short enough over which the perturber has not moved appreciably in the trajectory, i.e. when a collision gives the perturber some extra energy, a second collision occurs to take back that energy be fore the trajectory has been modified substantially.

For this reason, in Fig.4 we first identify the dominant width contributions. We see that velocities between $0.3\langle v\rangle$ and $0.9\langle v\rangle$ provide the dominant contributions, with the relevant IP ranging from about $4.4-7.5 \AA$ for the lowest velocity range and 1.5-3A for the highest velocity range. Then, in Figs. (5A) and 5(B) we plot $\left\{U_{a}(t, \rho, v) U_{b}^{\dagger}(t, \rho, v)-1\right\}$ vs. time $t$ (in attoseconds), with $U(t)$ the time evolution matrix for the upper $(a)$ and lower $(b)$ levels $^{3}$. In both cases this quantity stays

3) For long times we see a saturation, which simply means that the interaction is finished and 

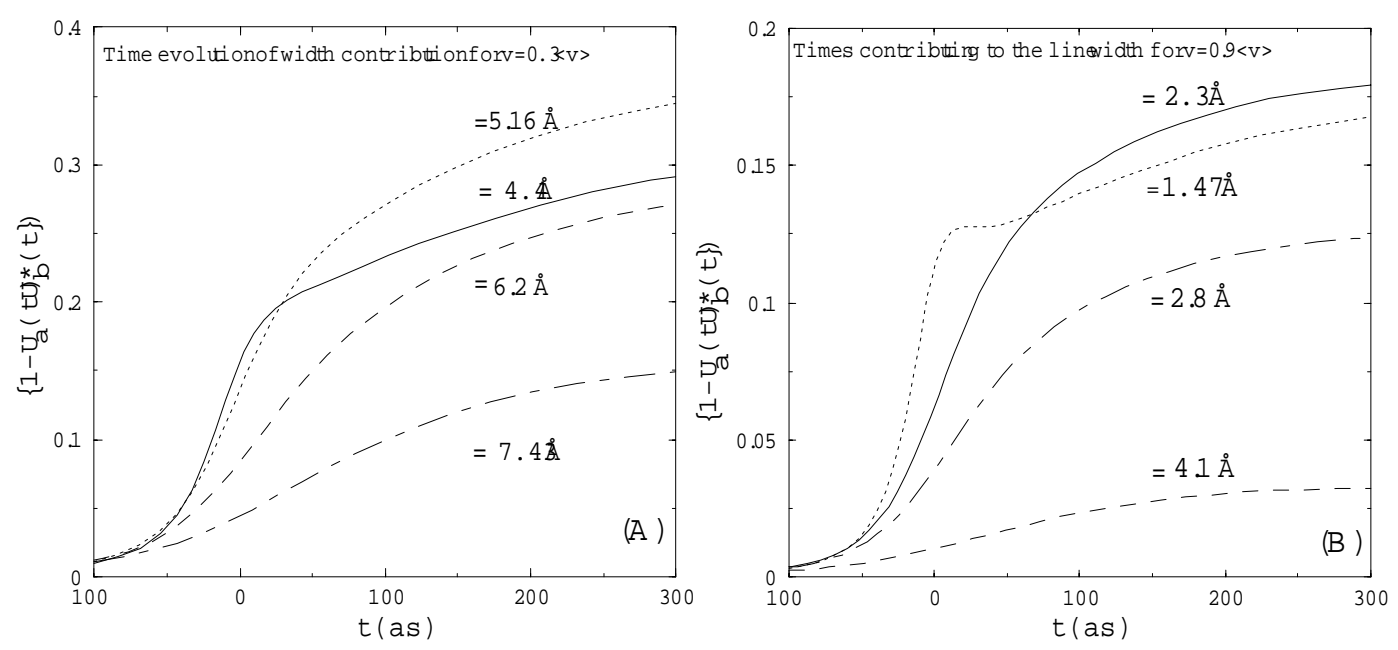

FIGURE 5. Time evolution of U-matrices

small, so that perturbation theory is valid, in agreement with the fact that the nonperturbative and perturbative(Coulomb Born) quantal methods agree and in contrast to the original NPSC. However, this means that essentially two collisions occur over the entire time interval, hence it is not true that collisions are as frequent as would be needed to justify neglecting the back reaction, i.e. lifetime in the $2 \mathrm{~s}$ and $2 \mathrm{p}$ states is not short compared to the trajectory time scale. This does not necessarily mean that back reaction is important and we note the qood agreement with QM although we could not prove that back reaction is not important.

\section{STATE OF SC}

NPSCII has evolved to a stage with few differences from the quantal approach. The differences are the de Broglie cutoff, resonances, which are an inherently quantal effect and back-reaction issues. Some work is underway on the first and third. Although in this specific case excellent agreement was found between NPSCII and $\mathrm{CC}$ calculations, it would be premature to conclude that this is always the case and more work is required on detailed comparisons. On the other hand the prospects for PR SC are very bleak. Essentially PR SC computes universal line SB functions $\mathrm{A}(+\mathrm{iB})$ which may be meaningless in many cases: First the width $\Phi$ is proportional to $\int d t_{1} e^{i \omega_{1} t_{1}} \int d t_{2} e^{i \omega_{2} t_{2}} \mathbf{d} \cdot \mathbf{E}\left(t_{1}\right) \mathbf{d} \cdot \mathbf{E}\left(t_{2}\right)$ where $\omega_{1}, \omega_{2}$ represent energy differences of states of the system in angular frequency units. In usual PR SC, the dipole matrix elements are taken outside the integral, which then factors into a purely atomic part and a part that depends on both perturber and atomic (via $\left.\omega_{1}, \omega_{2}\right)$ data. If one can set a $\rho_{\min }(v)$ such that all the WF is inside that cutoff(recall that the normal $n^{2} a_{0} / Z$ cutoff will not do), then this is fine in principle, but often useless in practice,

the U-matrices have become S-matrices. 
as the error bound is then much larger than what is presumably accurately computed. Otherwise, to effect this factorization, one needs to introduce the correction factors, which depend on the actual WFs, so that we can no longer have universal $\mathrm{A}$ and $\mathrm{B}$ functions. Hence in this specific example, although perturbation theory was valid, the standard $P R S C$ was not, since the reason perturbation theory was valid was the inapplicability of the long-range approximation (weakening of the interaction for IPs inside the WFE).

\section{CONCLUSIONS}

Both the SC and QM methods have come a long way, though more work is needed, particularly for dense plasmas, where both methods assume the asymptotic wavefunction, which in reality is a disordered state. Current efforts are directed to completing a detailed comparison with all available experimental data. Interfacing NPSCII to Cowan's code to make such comparisons easy is a first step. Regarding experiments, we have no grounds to question them, as outlined in [11]. However, a further check would be the simultaneous measurement of an isolated ion line and a line whose profile is determined by ions, for example the $L y_{\beta}$ or $H_{\beta}$ line of hydrogen or H-like Helium. Ideally we would also like to measure a Doppler-dominated line, so that we can be sure of electron density as well as the Doppler temperature. A match in the plasma parameters from TS and such independent line widths would be a very strong indication that the TS diagnostics are not in error.

\section{REFERENCES}

1. S.Alexiou, Phys.Rev.A.49, 106(1994);

2. S.Alexiou, Phys.Rev.Lett.75, 3406(1995);

3. S.Glenzer, N.I.Uzelac and H.J.Kunze, Phys.Rev.A45, 8795(1992); T.Wrubel et.al., Astron.Astrophys.306, 1028(1996).

4. S.Glenzer and H.J.Kunze, Phys.Rev.A.53, 2225(1996).

5. M.J.Seaton, J.Phys.B20, 6431(1987); M.J.Seaton, J.Phys.B21, 3033(1988).

6. H.R.Griem, Yu.Ralchenko and I.Bray, Phys.Rev.E.56 , 7186 (1997).

7. Yu.V.Ralchenko et.al., Phys.Rev.A59, 1890(1999).

8. N.Konjevic and W.L.Wiese, J.Phys.Chem.Ref.Data 19, 1307(1990).

9. H.R.Griem, Plasma Spectroscopy, Mc-Graw Hill, New York(1964), p.76.

10. See H.R.Griem, Spectral line Broadening by Plasmas, Academic, New York(1974); M.Baranger, in Atomic and Molecular Processes(D.R.Bates, editor), Academic, New York(1962), p.523; H.Griem and K.Shen, Phys.Rev.122, 1490(1962), p.1492 and Appendix; H.Griem, M.Blaha and P.Kepple, Phys.Rev.A19, 2421(1979), p.2423;

11. S.Alexiou, S.Glenzer and R.W.Lee, Phys.Rev.E.60 6238(1999).

12. H.Griem and Yu.Ralchenko, J.Quant.Spectrosc.Radiat.Transf.65, 287(2000).

13. S.Alexiou, R.W.Lee, S.Glenzer and J.Castor, JQSRT65, 15(2000). 